\title{
Predicting the Firmness of Apples using a Non-contact Ultrasonic Technique
}

\author{
Sangdae Lee ${ }^{1}$, Jeong-Gil Park ${ }^{1}$, Hyun-Mo Jeong ${ }^{2}$, Ki-Bok Kim ${ }^{3}$, Byoung-Kwan Cho ${ }^{1}$ * \\ ${ }^{1}$ Department of Biosystems Machinery Engineering, Chungnam National University, Daejeon 305-764, Korea \\ ${ }^{2}$ Division of Visual Design, Kyongbuk Science University, Chilgok 718-851, Korea \\ ${ }^{3}$ Center for Safety Measurement, Korea Research Institute of Standards and Science, Daejeon 305-340, Korea
}

Received: July 29 $9^{\text {th }}, 2013$; Revised: August $5^{\text {th }}, 2013$; Accepted: August $8^{\text {th }}, 2013$

\section{Abstract}

Purpose: Methods for non-destructive estimation of product quality have been reported in various industrial fields, but the application of ultrasonic techniques for the agricultural products of potatoes, pears, apples, watermelons, kiwis and tomatoes etc. have been rarely reported since the application of a contact-type ultrasonic transducer in agricultural products is very difficult. Therefore, this study sought to determine the firmness of apples using non-contact ultrasonic techniques. Methods: For this experiment, an ultrasonic experimental tester using a non-contact ultrasonic transducer was created, and a signal processing program was used to analyze the acquired ultrasonic reflected signal. Also, a universal testing machine was used to measure firmness parameters of the apples such as bioyield strength, a firmness factor, after the ultrasonic tests had been performed. Results: Six distance correction factors were calculated to obtain consistent values of ultrasonic properties regardless of the distance between the transducer and the surface of the subject. We developed prediction models of the bioyield strength using the distance correction factors. Conclusions: The optimum prediction model of the bioyield strength of apples using a non-contact ultrasonic technique was a multiple regression model $\left(\mathrm{R}^{2}=0.9402\right)$.

Keywords: Apple, Firmness, Non-contact ultrasonic technique, Universal testing machine

\section{Introduction}

Alongside the improved quality of life for many people and their increased incomes, purchase patterns for fruit are becoming diverse. Unlike quantity-oriented purchase patterns of the past, quality-based choice has become a recent trend. Also, as a greater variety of fruits became available since the opening of the domestic market to foreign produce, demand increased for the development of quality assessment techniques to ensure high standards of quality in the agricultural products to improve the international competitiveness of domestic products. Some domestic fruits, including apples and pears, are important strategic export items that require more extensive technical expertise and investment in quality control after the

\footnotetext{
*Comesponding author: Byoung-Kwan Cho

Tel: +82-42-821-6715; Fax: +82-42-823-6249

E-mail: chobk@cnu.ac.kr
}

harvest. Fruits are still living organisms even after they are harvested, and they continuously undergo physiological changes throughout the stages of distribution and storage. Therefore, a non-destructive quality assessment technique is necessary, and studies on the application of ultrasonic techniques that are closely related to the mechanical properties of fruits are being carried out (Kim et al., 2004).

The advantage of using an ultrasonic technique for the quality assessment of fruits is that the results of ultrasonic tests can be produced in real time, allowing for the automation of the system. Also, that technique can be easily formed into a measurement system, and there are no risk factors affecting the subjects of examination or the examiners. Since ultrasonic waves are sensitive to the structure of radio wave media and its elastic properties, it can provide information on the physical properties of an inside structure that cannot be known by other means. If 
a technique can be developed to determine important quality factors of fruits during the stages of sorting, packaging, storing and transportation, and to measure the firmness of the fruits (important for the prevention of degradation), then it will allow the whole fruit to be properly inspected and thereby contribute to increasing the income of farmers.

The existing method of using contacting ultrasonic techniques developed by other studies was difficult to apply on site during the sorting of fruit because it takes too much time to let the ultrasonic transducer make contact with the fruit because of the irregular sphericity of the fruit (Kim et al., 2004). To avoid additional time for manipulating the ultrasonic transducer and the fruit for proper contact in the measurement of firmness, a new concept for developing a firmness measurement system using an air-bonding ultrasonic transducer as a non-contact ultrasonic transducer emerged.

Many researchers have investigated the relationship between ultrasonic parameters and the firmness of agricultural products (Yamamoto et al., 1980; Duprat et al., 1997; Mizrach et al., 1998; Kim et al., 2004). Finney (1970) showed good correlation between the extent of the ripeness of fruits and their elastic modulus. Garret and Furry (1972) measured mechanical properties such as Young's modulus, tissue density, and the Poisson's ratio of apples using various wave propagation velocities. Clark (1975) reported that the decay time of sound waves crossing watermelons increase with a watermelon's ripeness. Mizrach et al. (1989) performed preliminary experiments to calculate velocities of wave propagation, attenuation rates, and reflection properties of tissue specimens of some fruits and vegetables. He reported that changes in the sound velocity of fruits and vegetables might be used for ripeness classification in some products. Lu and Huang (2000) investigated the effect of the contact force of an ultrasonic transducer on the ultrasonic attenuation measurement of radishes. Lee at al. (2010) developed a $500 \mathrm{kHz}$ noncontact ultrasonic transducer to measure the fruit firmness. Lee et al. (2013) investigated the feasibility of using a non-contact ultrasonic technique to measure the firmness of apple and peach.

The purpose of this study was to establish a method of compensating the values of ultrasonic reflection parameters caused by irregular distances between a non-contacting ultrasonic transducer and the surface of the fruit. We also aimed to develop firmness-predicting models based on the analysis of correlations between non-contacting ultrasonic parameters and bioyield strength, a firmness factor.

\section{Materials and Methods}

The cultivars of apples used in this study, YouKou and Fuji, were harvested from one apple tree each on an ordinary farm. Apples were brought to be tested as soon as they were harvested and selected according to the absence of blemishes and defects. They were kept in the laboratory at a temperature of $21^{\circ} \mathrm{C}$ and relative humidity of $75 \%$. Thirty apples were sampled at a time at 3-day intervals totaling 7 tests on YouKou and 9 tests on Fuji.

The non-contacting ultrasonic measurement system for the study of ultrasonic properties of apples were composed with an ultrasonic pulser/receiver (HIS-2, AC $100 \sim 240 \mathrm{~V} \pm 10 \%, 100$ VA max.) shown in Figure 1, a non-contacting ultrasonic transducer (NCT55, Ultran Inc., USA) with a mean frequency of $0.5 \mathrm{MHz}$, a ultrasonic transducer anchoring device and distance measuring device, an oscilloscope with a sampling rate of $1 \mathrm{GHz}$ and a computer. MATLAB software (MathWorks Inc., USA) was used for developing prediction models.

Those apples on which reflection tests were carried out after the ultrasonic test were again used for UTM (universal testing machine) plate compression tests to measure bioyield strength, a firmness factor, using a loading rate of 5 $\mathrm{mm} / \mathrm{min}$ as specified in the ASABE Standard.

After removing the interruption of the initial excitation signal on the ultrasonic transducer, ultrasonic signals were measured using a non-contacting reflection method adjusting the distance between the surface of the apple and the transducer in intervals of $5 \mathrm{~mm}$ for YouKou and 3 $\mathrm{mm}$ for Fuji within the range of $0 \sim 25 \mathrm{~mm}$ and $0 \sim 15 \mathrm{~mm}$, respectively. For the measurement and analysis of noncontacting ultrasonic reflection signals, the largest amplitude and TOF (time of flight) values of the first and second reflection signals were selected in the time domain and

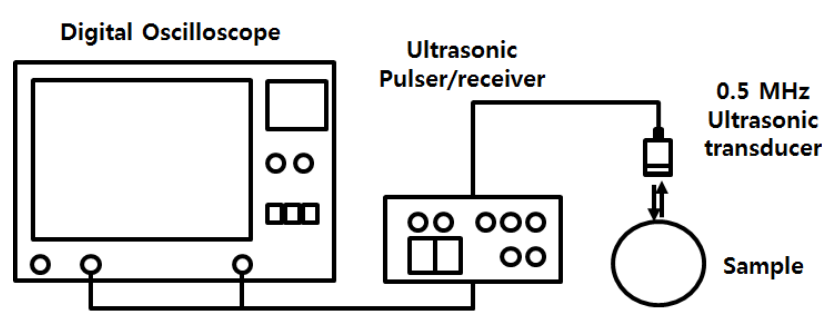

Figure 1. Non-contact ultrasonic measurement system. 
the first and second magnitude. PSD (power spectral density) values of the first and second reflection signals were selected in the frequency domain. Combinations of 10 total parameters were applied to the development of the bioyield strength-predicting model after the distance correction.

To compensate for the irregularities of ultrasonic signals caused by the varying distances between the non-contacting ultrasonic transducer and the surface of the fruit, five types of industrial materials, as rubber, aluminum, Teflon, wood, and steel, were selected.

Ultrasonic signals were measured using a reflection method, adjusting the distance between the surface of the sample and the ultrasonic transducer in $5 \mathrm{~mm}$ intervals within the range of $0 \sim 25 \mathrm{~mm}$. Amplitudes of the first and second measured reflection signals were calculated using the signal processing program developed in this study. Results were analyzed through fast Fourier transform (FFT) in the frequency domain and the energy ratio of the frequency band was deduced. Combinations of 10 total ultrasonic parameters were used to determine the distance correction factor. Then, the prediction models were developed using the distance correction factors.

\section{Results and Discussion}

\section{Distance correction factor}

Figure 2 shows reflection signals off the rubber, one of the materials used in the test. As shown in Figure 2, the
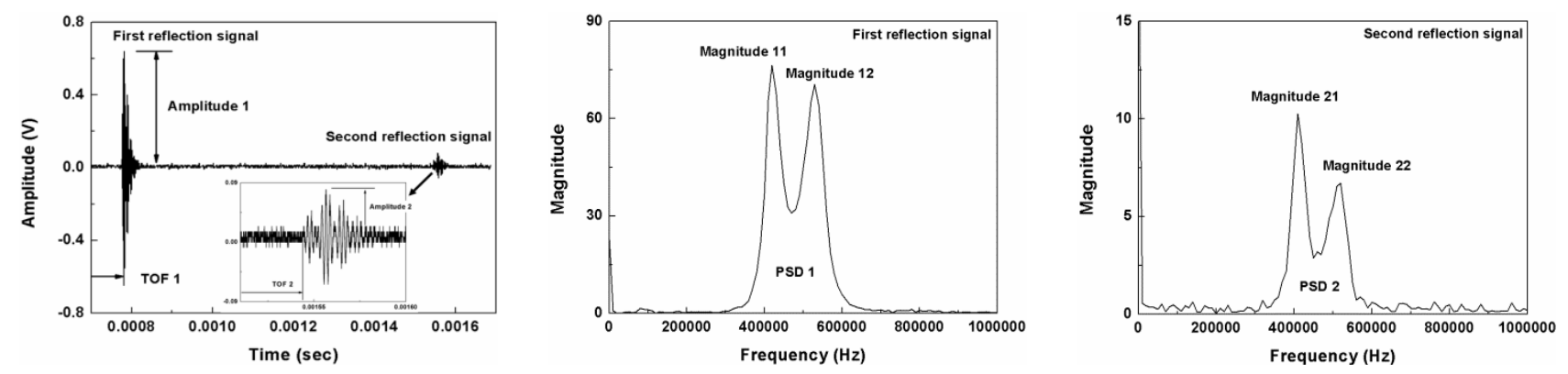

Figure 2. Typical reflected signal of the sample material (rubber) at the initial position.

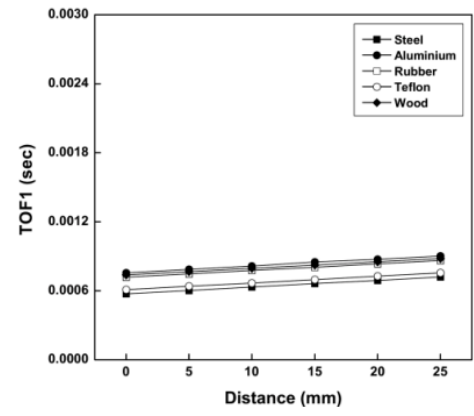

(a) TOF1

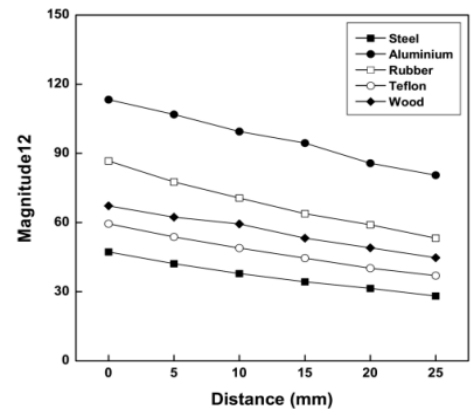

(d) Magnitude12

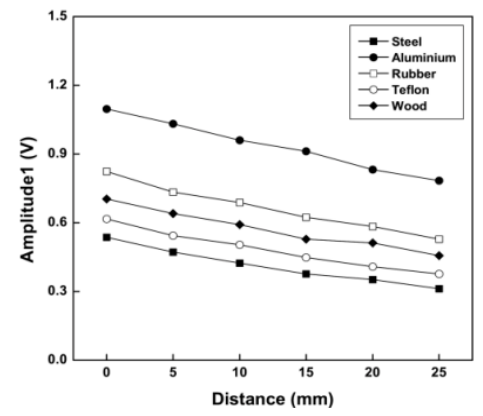

(b) Amplitude1

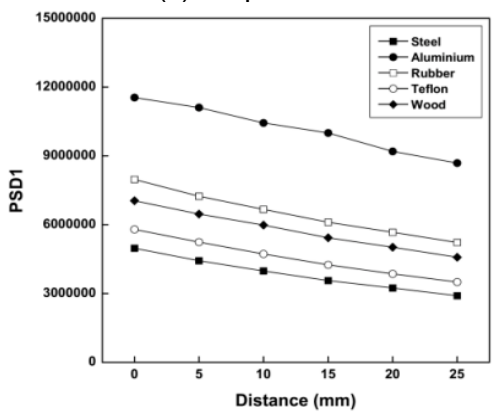

(e) PSD1

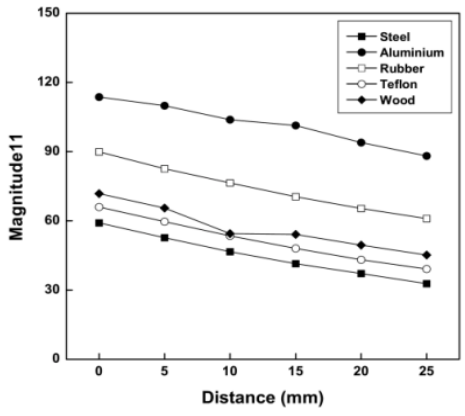

(c) Magnitude1

Figure 3. Ultrasonic parameters of first reflection signal according to the distance between the ultrasonic transducer and the surface of objects. 
largest amplitude of the first and second reflection signals was observed in the time domain. Also, the result of FFT showed a significantly larger magnitude of the first reflection signal in the frequency domain than the magnitude of the second reflection signal in the time domain. These results suggest that the dampening effect by air is greater on the second reflection signal.

Figure 3 shows the changing values of the ultrasonic parameters of first reflection signal off the surface of the sample according to distance. Values increased consistently as the distance increased only in the TOF of the first and second reflection signals. For the other eight parameters, values tended to decrease as distance increased.

Since the dampening effect of air differed depending on the distance during the measurement using the non-contacting ultrasonic transducer, results of an analysis using the actual measurement data will have distance-dependent errors. Therefore, a correction has to be done to obtain consistent values of ultrasonic properties, regardless of the distance between the ultrasonic transducer and the surface of the subject. A variety of distance correction methods were tried on the 10 ultrasonic parameters, including the amplitude of the received signals, TOF, magnitude and PSD. Six distance correction factors were identified as the formulas 1 to 6 and shown in Figure 4 .
Detail information is provided in previous publications (Lee et al. 2010, Lee et al. 2013). Every correction factors had no significant difference $(\mathrm{P}>0.05)$ according to the distance between the ultrasonic transducer and the apple.

$$
\begin{aligned}
& \text { Factor }_{1}=\left(\mathrm{amp}_{2} / \mathrm{amp}_{1}\right) /\left(\mathrm{TOF}_{2} / \mathrm{TOF}_{1}\right) \\
& \text { Factor }_{2}=\left(\mathrm{mag}_{21} / \mathrm{mag}_{11}\right) /\left(\mathrm{TOF}_{2} / \mathrm{TOF}_{1}\right) \\
& \text { Factor }_{3}=\left(\mathrm{mag}_{22} / \mathrm{mag}_{12}\right) /\left(\mathrm{TOF}_{2} / \mathrm{TOF}_{1}\right) \\
& \text { Factor }_{4}=\left(\mathrm{mag}_{12} / \mathrm{mag}_{11}\right) /\left(\mathrm{TOF}_{2} / \mathrm{TOF}_{1}\right) \\
& \text { Factor }_{5}=\left(\mathrm{mag}_{22} / \mathrm{mag}_{21}\right) /\left(\mathrm{TOF}_{2} / \mathrm{TOF}_{1}\right) \\
& \text { Factor }_{6}=\left(\mathrm{PSD}_{2} / \mathrm{PSD}_{1}\right) /\left(\mathrm{TOF}_{2} / \mathrm{TOF}_{1}\right)
\end{aligned}
$$

where $\mathrm{amp}_{1}$ is max. amplitude of the first reflection signal, $\mathrm{amp}_{2}$ is max. amplitude of the second reflection signal, $\mathrm{TOF}_{1}$ is time of flight of the first reflection signal, $\mathrm{TOF}_{2}$ is time of flight of the second reflection signal, mag $_{11}$ is first magnitude of the first reflection signal, $\operatorname{mag}_{12}$ is second magnitude of the first reflection signal, mag $_{21}$ is first magnitude of the second reflection signal, $\operatorname{mag}_{22}$ is Second

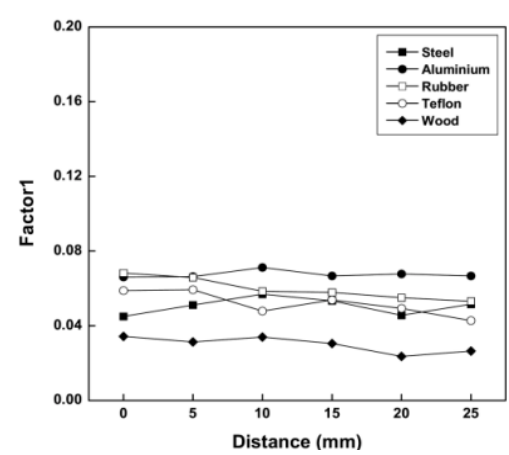

(a) Factor1

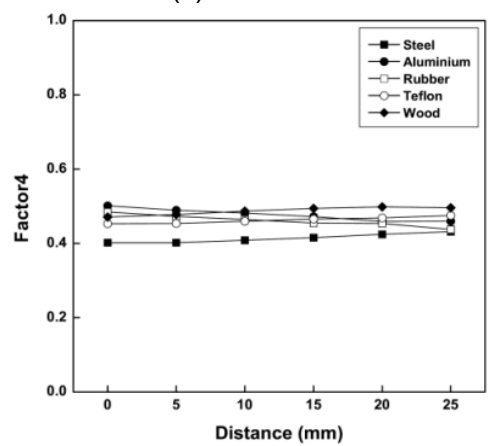

(d) Factor4

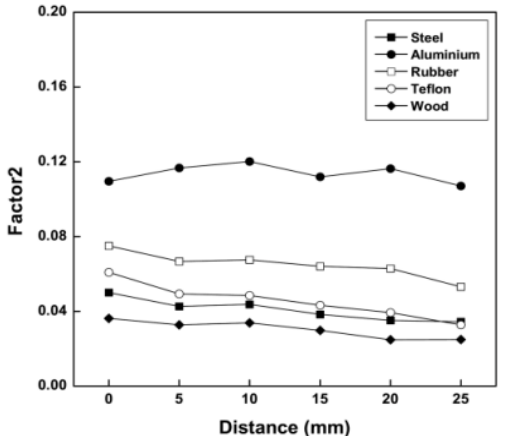

(b) Factor2

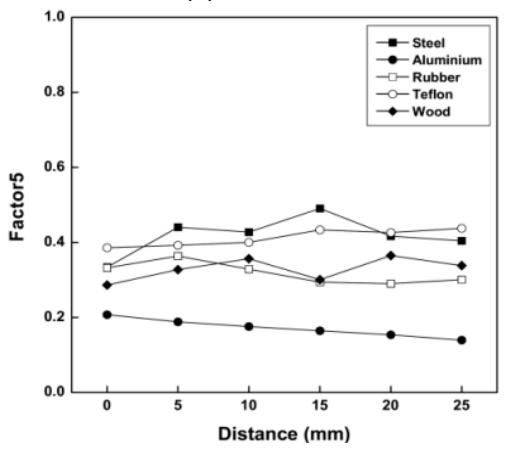

(e) Factor5

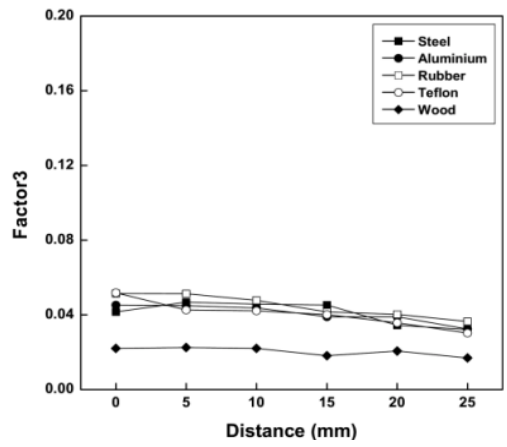

(c) Factor3

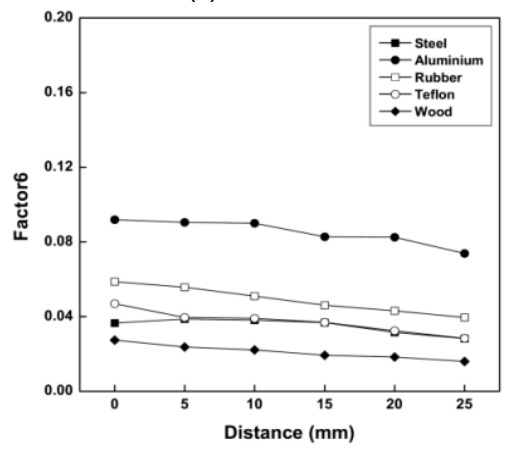

(f) Factor6

Figure 4. Corrected ultrasonic factors considered with the distance effect. 
magnitude of the second reflection signal, $\mathrm{PSD}_{1}$ is Power spectrum density of the first reflection signal and $\mathrm{PSD}_{2}$ is Power spectrum density of the second reflection signal.

\section{Models to estimate the firmness of apples}

The distance correction factors determined above were applied here. Since the distance between the ultrasonic transducer and the surface of the apple is expected to be approximately within $10 \sim 15 \mathrm{~mm}$ when the measurement is taken using a non-contacting ultrasonic transducer, the parameters obtained at 5 15 mm for YouKou and 6 12 $\mathrm{mm}$ for Fuji were used in the development of the bioyield strength-predicting model by applying the distance correction factors already found while the interruption of initial excitation signals to the ultrasonic transducer was kept at a minimum. Figure 5 shows the force-deformation curve

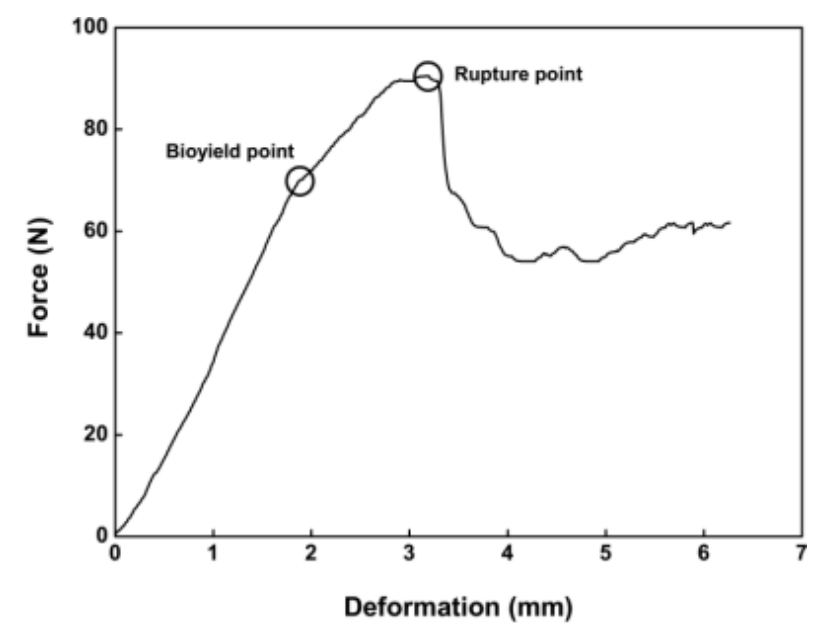

Figure 5. Force-deformation curve of an apple. of the apple evaluated using a compression test, and the bioyield strength, a firmness factor, was measured on this curve.

\section{Multiple linear regression model}

A multiple linear regression model was developed using the bioyield strength of the apple and the distance correction factors of ultrasonic reflection signals. The prediction model developed for each variety showed no significant difference in their determination coefficients, which were 0.9312 for YouKou and 0.8949 for Fuji as shown in Table 1. The multiple linear regression model could be used to make relatively accurate predictions of the bioyield strength of an apple.

\section{Principal component analysis model}

In principal component analysis, statistic results can be unstable if the problem of multi-colinearity, which is interdependence between parameters, is not taken into account. Hence correlations between distance correction factors for each variety of apple were carried out, and results indicated that there was no correlation between the distance correction factors. These distance correction factors could be used in the analysis of principal components. Results of principal component analysis indicated that $99 \%$ of the variation of the entire data was found in principal components 1 to 4 among all the principal components. This indicates that a bioyield strength-predicting model can be established with principal components 1 to 4 only. The bioyield predicting model developed using principal components 1 to 4 produced the results shown in Table 2 .

\begin{tabular}{|c|c|c|c|c|c|c|c|c|}
\hline \multirow{2}{*}{ Cultivar } & \multicolumn{7}{|c|}{ BS $=a \times$ Factor $_{1}+b \times$ Factor $_{2}+c \times$ Factor $_{3}+d \times$ Factor $_{4}+e \times$ Factor $_{5}+f \times F_{\text {Factor }}+g$} & \multirow{2}{*}{$r^{2}$} \\
\hline & a & $b$ & $c$ & $d$ & e & $f$ & $g$ & \\
\hline YouKou & 826.432 & -444.379 & 1154.787 & 193.918 & -162.153 & -1540.052 & 188.724 & 0.9402 \\
\hline Fuji & -499.518 & 2100.376 & -1266.254 & -56.292 & 116.502 & -24.691 & 102.989 & 0.8949 \\
\hline
\end{tabular}

Note : BS $=$ Bioyield Strength, Factor 1 6 = Distance correction factors

Table 2. Principal component regression equation for bioyield strength of the apples

\begin{tabular}{ccccccc} 
& \multicolumn{7}{c}{$Y=a \times P C_{1}+b \times P C_{2}+c \times P C_{3}+d \times P C_{4}+e$} & & $r^{2}$ \\
\cline { 2 - 6 } Cultivar & $a$ & $b$ & $c$ & $d$ & 158.492 & 0.5939 \\
YouKou & -83.390 & -192.236 & -273.217 & -456.980 & 77.992 & 0.6082 \\
\hline Fuji & 390.566 & -132.888 & -259.398 & -237.077 & \\
\hline
\end{tabular}

Note : $\mathrm{Y}=$ Bioyield strength, $\mathrm{PC}_{1}=1^{\text {st }}$ principal component, $\mathrm{PC}_{2}=2^{\text {nd }}$ principal component, $\mathrm{PC}_{3}=3^{\text {rd }}$ principal component, $\mathrm{PC}_{4}=$ $4^{\text {th }}$ principal component 
Table 3. Correlation coefficient between measured bioyield strength of apple and predicted one by Artificial Neural Network model

\begin{tabular}{ccccccccc} 
Cultivar & \multicolumn{8}{c}{ Node No. } \\
\cline { 2 - 9 } & 1 & 3 & 5 & 7 & 9 & 11 & 13 & 15 \\
YouKou & 0.9435 & 0.9102 & 0.8596 & 0.2728 & 0.3761 & 0.3775 & 0.2815 & -0.2268 \\
Fuji & 0.9023 & 0.6771 & 0.7352 & 0.3965 & 0.3432 & 0.3956 & 0.3022 & 0.2081 \\
\hline
\end{tabular}

Analysis of the principal component regression model developed in this way showed that the determination coefficient values of the model were generally lower than those of the multiple regression model and the values were 0.5939 for YouKou and 0.6082 for Fuji. The principal component analysis showed, unlike the multiple linear regression model, that the determination coefficient value for Fuji was slightly higher than that of YouKou.

\section{Artificial neural network model}

The structure of a neural network model for the prediction of the firmness of apples was constructed with one hidden layer considering the frequency and speed of learning, the convergence rate, the results of output and six distance correction factors, which were analyzed as the input patterns of the input layer, were used. The number of nodes in the hidden layer for each variety of apple was incremented in 2-step intervals within a range of $1 \sim 15$ as shown in Table 3. For each number of nodes, analysis was performed by carrying out a number of tests to find the optimum value. The target error for YouKou in the learning process was set to 0.009 , learning rate to 0.001 and momentum to 0.95 . For Fuji apples, the values were set at 0.05 for target error and 0.001 and 0.95 for the learning rate and momentum, respectively. The learning process was repeated until the error was below the target. The neural network built in this study used the tangent sigmoid function as the transfer function from input layer to hidden layer and the pure linear function as the transfer function from hidden layer to output layer. The results of the learning process showed high correlation coefficients of YouKou and Fuji, where YouKou was 0.9435 and Fuji was 0.9023 when the number of nodes was 1 . However, the results of the neural network analysis showed significant differences between correlation coefficients as the number of nodes, learning rate and error target changed. There may be difficultly in applying neural network analysis to the system because many variables arise in the course of finding the optimum value through repeated learning processes.

Comparative studies on bioyield strength-predicting models developed from these three different methods of analysis indicated the neural network model to be the best, but not with significant differences from the other methods. If prediction accuracy between prediction models is not significant, then the multiple regression model was believed to be the appropriate choice since it provides relatively simpler means for predicting the bioyield strength.

\section{Conclusions}

In this study, we measured ultrasonic parameters for apples for each distance between a non-contact ultrasonic transducer and the surface of the apples for on-line quality determination, and made corrections so that ultrasonic parameters were not affected by differences in the distance. We developed prediction models (MLR, PCR, ANN) of the bioyield strength using the distance correction factors. The optimum prediction model of the bioyield strength of apples using a non-contact ultrasonic technique was a multiple linear regression model $\left(\mathrm{R}^{2}=0.9402\right)$.

\section{Conflict of Interest}

The authors have no conflicting financial or other interests.

\section{References}

ASABE Standard S368.3 MAR95, 1996.

Clark, R. L. 1975. An investigation of the acoustical properties of watermelon as related to maturity. ASABE Paper No. 75-6004. st. Joseph, MI.

Duprat, F., M. Grotte, E. Pietri and D. Loonis. 1997. The acoustic impulse response method for measuring the overall firmness of fruits. Journal of Agricultural Engineering Research. 66(4):251-259.

Grrett, R and R. Flurry. 1972. Velocity of sonic pulse in apples. Transaction of the ASAE 11(2):94-97.

Kim, K. B., H. M. Jung, M. S. Kim and G. S. Kim. 2004. 
Evaluating of fruit firmness by ultrasonic measurement. Key Engineering Materials. 270-273:1049-1054.

Finney, E. E., 1970. Mechanical resonance within 'Red Delicious' apples and its relation to fruits texture. Transaction of the ASAE. 13(2):177-180.

Lee, S., T. H. Ha, K. B. Kim and M. S. Kim. 2010. Study on non-contact ultrasonic transducer for measurement of fruit firmness. Journal of Biosystems Engineering. 35(3):189-196 (In Korean).

Lee, S., M. Hasegawa, K. B. Kim, J. G. Park and B. K. Cho. 2012. Evaluation of the firmness measurement of fruit by using a non-contact ultrasonic technique. Journal of the Faculty of Agriculture Kyushu University.
58(1):103-108.

Mizrach, A., N. Galili and G. Rosenhouse. 1989. Determination of fruits and vegetables properties by ultrasonic excitation. Transaction of the ASAE. 32(6):2053-2059. Moghavvemi, M., M. O. Faruque, A. Mizrach, U. Flitsanov, Z. Schmilovitch and Y. Fuchs. 1998. Determination of mango physiological indices mechanical wave analysis. Postharvest Biology and Technology. 16:179-186.

Yamamoto, H., M. Iwamoto and S. Haginuma. 1980. Acoustic impulse response method for measuring natural frequency of intact fruits and preliminary applications to internal quality evaluation of apples and watermelons. Journal of Texture Studies. 11:117-136. 